\title{
Camera Calibration and Scene Modeling from Arbitrary Parallelograms Imposing the Multiview Constraints
}

\author{
Jae-Hean Kim \\ gokjh@etri.re.kr \\ Bon-Ki Koo \\ bkkoo@etri.re.kr
}

\author{
Content Research Division \\ Electronics and Telecommunications \\ Research Institute \\ Daejeon, Republic of Korea
}

\begin{abstract}
This paper proposes a novel framework using geometric information on parallelograms for camera calibration and scene modeling. The proposed method solves the problem linearly with factorizing a measurement matrix into the camera and plane parameters. The measurement matrix contains canonic planar homographies between camera views and planes on which arbitrary parallelograms lie. Since the factorization based approaches recover all camera poses simultaneously, the consistency of rigid transformations among cameras can be ensured. The previous parallelogram-based approaches solving the problem linearly cannot guarantee this consistency because they are based on the infinite homographies extracted from the individual pairs of camera images. The proposed method is more useful than the previous factorization based approach using parallelepipeds because parallelograms are more basic scene elements. The results of the experiments with real outdoor images are presented to demonstrate the feasibility of the proposed method.
\end{abstract}

\section{Introduction}

This paper addresses the problem of recovering camera parameters and scene model linearly and simultaneously from the images acquired with uncalibrated cameras. This process is known as camera self-calibration. A Euclidean reconstruction from the self-calibration is not possible without any prior information on cameras or on the scene to be recovered. According to the a priori information assumed to be given, there have been many approaches concerning the self-calibration algorithm.

Self-calibration approaches using only the constraints on the internal parameters are known as auto-calibration and have been widely investigated. These approaches provide

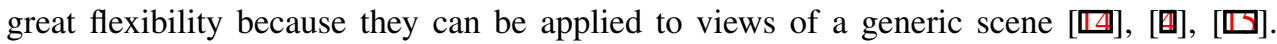
However, to acquire stable estimation results, a large number of images is usually necessary. Algorithms using the constraints from the camera motions have been also suggested

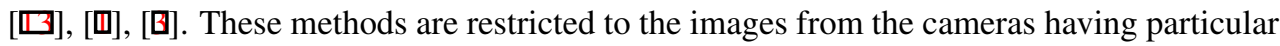
motions such as pure translation or pure rotation.

There have been many methods to use the constraints from scene geometry: vanishing

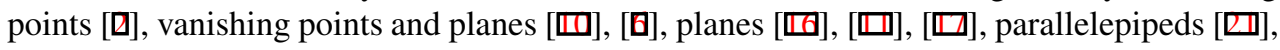


parallelograms $[\boldsymbol{\theta}],[\mathrm{D}],[\mathrm{G}],[\mathrm{Q}]$. Due to the geometric information from the scene, these approaches can give more stable estimation results without lengthy image sequences.

In the images captured in man-made environments, there are many primitives giving the geometric information. Among primitives, parallelograms and parallepipeds are frequently present in the scene, such as the architecture. The scene's affine structure is embedded in them and their Euclidean structure related to the shape can be used to upgrade the affine structure to the metric one.

Wilczkowiak et al. suggested an elegant formalism using parallelepipeds of which at least the six vertices are visible in views [ $\square]$. This method is a factorization-based approach that computes the camera parameters and scene structure parameters linearly and simultaneously in one step. Due to the multiview constraints imposed on a factorization approach, it is possible to obtain the consistency of rigid transformations among cameras. If this consistency is not guaranteed, the estimation accuracy can be degraded. It has been known that all image measurements should be used simultaneously to obtain optimal estimates as in the factorization approach [四].

The primitive that can give full affine information is not only a parallelepiped. Parallelograms are more general primitives in man-made environments. Since pairs of parallelograms do not always form two faces of a parallelepiped, the methods using parallelograms are more flexible in use [ $[\mathbb{Z}]$, [ם]. However, the previous linear approaches using parallelograms cannot guarantee the above consistency because they are based on the information extracted from the individual pairs of camera images and should combine the individual results when more than two views are given.

In this paper, we suggest a factorization-based framework that utilizes parallelograms in general position and ensures consistent results. The general position means that the parallelograms need not to be the part of a parallelepiped. One measurement matrix includes all image measurement in all views and is factorized into the camera and plane parameters. The contributions of this paper concerns the formulation of the measurement matrix from parallelograms and the computation of the scale factors necessary for the factorization.

Many methods have been introduced to use geometric constraints of a scene and multiview constraints giving the consistent estimates. The works very similar to that proposed in this paper were presented by Sturm [ㅁ] and Ueshiba and Tomita [ $\mathbb{}$ ] $]$. These methods adopt factorization-based approach to compute camera parameters and plane parameters. However, these methods require known metric structure of the planes. Rother et al. showed that it is also possible to obtain projective reconstruction linearly with the points on planes using factorization $[\square]$. This method uses inter-image homography induced by the planes. However, a certain plane is required as a reference and this significantly affects the accuracy. Malis and Cipolla proposed a self calibration algorithm using images of a plane of which the structure is unknown $[\square]$. Although this method is not categorized as a factorization-based approach and is a non-linear iterative approach, it imposes multiview constraints using all inter-image homographies to increase estimation accuracy. 


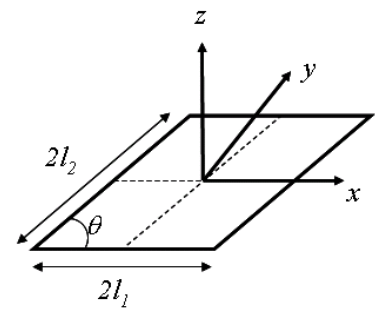

Figure 1: Parameterization of a parallelogram.

\section{Preliminaries}

\subsection{Camera Parameterization}

The camera is represented using pinhole model. The projection model of a point $\mathbf{X}$ in $3 \mathrm{D}$ world coordinate system to a point $\mathbf{x}$ in 2D image is expressed as follows:

$$
\mathbf{x} \cong \mathbf{K}\left[\begin{array}{ll}
\mathbf{R} & \mathbf{t}
\end{array}\right] \mathbf{X} \cong \mathbf{M X},
$$

where $\mathbf{x}$ and $\mathbf{X}$ are homogeneous coordinates of the points and ' $\cong$ ' indicates equality up to scale. The rotation matrix $\mathbf{R}$ and the vector $\mathbf{t}$ represent camera's orientation and position. The $3 \times 3$ matrix $\mathbf{K}$ is the camera calibration matrix [ $\mathbf{\theta}]$.

$$
\mathbf{K}=\left[\begin{array}{ccc}
f_{u} & s & u_{0} \\
0 & f_{v} & v_{0} \\
0 & 0 & 1
\end{array}\right]
$$

The $3 \times 4$ matrix $\mathbf{M}$ encapsulates the camera intrinsic and extrinsic parameters. The image of the absolute conic (IAC) $\omega$ is simply related to the intrinsic parameters by $\omega=$ $\mathbf{K}^{-T} \mathbf{K}^{-1}$.

\subsection{Parallelogram Parameterization}

A parallelogram is defined by 9 parameters: six extrinsic parameters describing its orientation and position and three intrinsic parameters describing its Euclidean shape: two dimension parameters (edge lengths $l_{1}$ and $l_{2}$ ) and one angle between edges $(\theta)$. These intrinsic parameters are illustrated in Fig. 1 . The parallelogram may be represented by a $2 \times 2$ matrix $\overline{\mathbf{L}}$ :

$$
\overline{\mathbf{L}}=\left[\begin{array}{cc}
l_{1} & l_{2} \cos \theta \\
0 & l_{2} \sin \theta
\end{array}\right] .
$$

The matrix $\overline{\mathbf{L}}$ represents the parallelogram's shape (intrinsic paramter). A vertex $\tilde{\mathbf{x}}_{\mathbf{s}} \equiv$ $( \pm 1, \pm 1)^{T}$ of the canonic square is mapped, by $\overline{\mathbf{L}}$, to a vertex of parallelogram on its supporting plane.

Let $\mathbf{x}_{\mathbf{s}}$ be homogeneous coordinates of vertex of the canonic square. Using this representation, the vertex of parallelogram on the world coordinate system is represented as follows:

$$
\begin{aligned}
\mathbf{X} & \cong\left[\begin{array}{cc}
\overline{\mathbf{S}} & \mathbf{v} \\
\mathbf{0}^{T} & 1
\end{array}\right]\left[\begin{array}{cc}
\overline{\mathbf{L}} & \mathbf{0} \\
\mathbf{0}^{T} & 1
\end{array}\right]\left[\begin{array}{c}
\tilde{\mathbf{x}}_{\mathbf{S}} \\
1
\end{array}\right] \\
& \cong \mathbf{N x}_{\mathbf{s}},
\end{aligned}
$$




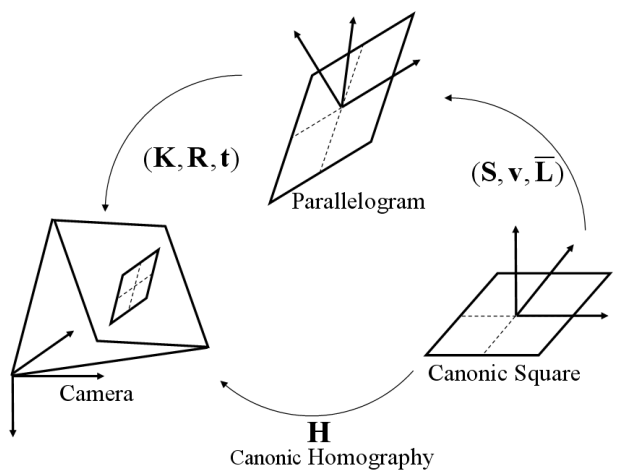

Figure 2: The projection of the canonic square onto the vertices of the imaged parallelograms.

where $\overline{\mathbf{S}}$ is the $3 \times 2$ submatrix consisting of the first two columns of a rotation matrix $\mathbf{S}$ representing the parallelogram's orientation and a vector $\mathbf{v}$ is its position.

\section{Projections of Parallelograms}

\subsection{Canonic Homography}

Consider the projection of a parallelogram's vertices into a camera image plane. Using results from Section 2, the projection of the corresponding vertex in the image is:

$$
\begin{aligned}
& \mathbf{x} \cong \mathbf{K}\left[\begin{array}{ll}
\mathbf{R} & \mathbf{t}
\end{array}\right]\left[\begin{array}{ll}
\overline{\mathbf{S}} \overline{\mathbf{L}} & \mathbf{v} \\
\mathbf{0}^{T} & 1
\end{array}\right] \mathbf{x}_{\mathbf{S}} \\
& =\mathbf{M N x}_{\mathbf{s}} \\
& =\mathbf{H x}_{\mathbf{s}} \text {. }
\end{aligned}
$$

The matrix $\mathbf{H}$ will be called the canonic homography. It represents a perspective projection that maps the vertices of the canonic square onto the vertices of the imaged parallelograms. This is illustrated in Fig 2. Given image points for four vertices, the canonic homography can be computed up to scale, even though we do not know prior knowledge on intrinisic or extrinisic parameters. Our calibration algorithms are based on the link between the canonic homography and the camera's and parallelogram's intrinsic and extrinsic parameters.

\subsection{Measurement Matrix of Homographies}

Let us now consider the case where $n$ parallelograms are seen by $m$ cameras. Let $\tilde{\mathbf{H}}_{i}^{j}$ be the estimation result of the canonic homography associated with the projection of the $j$ th parallelogram in the $i$ th camera and $\lambda_{i}^{j}$ a scale factor such that the following equality can be 
written:

$$
\begin{aligned}
\lambda_{i}^{j} \tilde{\mathbf{H}}_{i}^{j} & =\mathbf{K}_{i}\left[\begin{array}{ll}
\mathbf{R}_{i} & \mathbf{t}_{i}
\end{array}\right]\left[\begin{array}{cc}
\overline{\mathbf{S}}^{j} \overline{\mathbf{L}}^{j} & \mathbf{v}^{j} \\
\mathbf{0}^{T} & 1
\end{array}\right] \\
& =\mathbf{M}_{i} \mathbf{N}^{j} \\
& =\mathbf{H}_{i}^{j} .
\end{aligned}
$$

We may gather the estimated canonic homography for all $m$ cameras and $n$ parallelograms into the following single matrix:

$$
\tilde{\mathbf{W}}=\left[\begin{array}{ccc}
\tilde{\mathbf{H}}_{1}^{1} & \cdots & \tilde{\mathbf{H}}_{1}^{n} \\
\vdots & \ddots & \vdots \\
\tilde{\mathbf{H}}_{m}^{1} & \cdots & \tilde{\mathbf{H}}_{m}^{n}
\end{array}\right]
$$

The matrix $\tilde{\mathbf{W}}$ will be called the measurement matrix. When the scale factors are recovered, the measurement matrix can be factorized as follows:

$$
\left[\begin{array}{ccc}
\lambda_{1}^{1} \tilde{\mathbf{H}}_{1}^{1} & \ldots & \lambda_{1}^{n} \tilde{\mathbf{H}}_{1}^{n} \\
\vdots & \ddots & \vdots \\
\lambda_{m}^{1} \tilde{\mathbf{H}}_{m}^{1} & \ldots & \lambda_{m}^{n} \tilde{\mathbf{H}}_{m}^{n}
\end{array}\right]=\left[\begin{array}{ccc}
\mathbf{H}_{1}^{1} & \ldots & \mathbf{H}_{1}^{n} \\
\vdots & \ddots & \vdots \\
\mathbf{H}_{m}^{1} & \ldots & \mathbf{H}_{m}^{n}
\end{array}\right]=\left[\begin{array}{c}
\mathbf{M}_{1} \\
\vdots \\
\mathbf{M}_{m}
\end{array}\right]\left[\begin{array}{lll}
\mathbf{N}^{1} & \ldots & \mathbf{N}^{n}
\end{array}\right] .
$$

However, since the canonic homographies are obtained up to scale, the measurement matrix cannot be factorized as its current form.

\section{Parameter Estimation}

\subsection{Rescaling Measurement Matrix}

In this section, we will describe how to obtain the scale factors mentioned in Section 3.2. Let $\pi^{j}$ be the supporting plane for the $j$ th parallelogram. Assume that the plane $\pi^{j}$ induces an inter-image homography $\mathbf{A}_{i k}^{j}$ from the $k$ th to the $i$ th camera image. This inter-image homography can be represented by canonic homograpies as $\mathbf{A}_{i k}^{j}=\mathbf{H}_{i}^{j}\left(\mathbf{H}_{k}^{j}\right)^{-1}$. Let $\pi^{l}$ be the supporting plane for another $l$ th parallelogram and $\mathbf{A}_{i k}^{l}$ be the homography induced by $\pi^{l}$. Composing $\mathbf{A}_{i k}^{l}$ with the inverse of $\mathbf{A}_{i k}^{j}$ yields a relative homography represented by $\mathbf{B}_{i k}^{j l}=\left(\mathbf{A}_{i k}^{j}\right)^{-1} \mathbf{A}_{i k}^{l}$, which map a point from the $k$ th camera image onto the same image. This relative homography is known to be a planar homology having the form $\mathbf{B}_{i k}^{j l}=\mathbf{I}+\mathbf{a b}^{T}$, where $\mathbf{a}$ and $\mathbf{b}$ are arbitrary 3-vectors [ $\mathbb{} \mathbf{Z}]$. This means that $\mathbf{B}_{i k}^{j l}$ has an eigenvalue 1 and the multiplicity of that eigenvalue is two.

The relative homography can be computed up to scale from the canonic homographies, which are also up to scale, as follows:

$$
\begin{aligned}
\tilde{\mathbf{B}}_{i k}^{j l} & =\tilde{\mathbf{H}}_{k}^{j}\left(\tilde{\mathbf{H}}_{i}^{j}\right)^{-1} \tilde{\mathbf{H}}_{i}^{l}\left(\tilde{\mathbf{H}}_{k}^{l}\right)^{-1} \\
& =\left(\lambda_{i}^{j} \lambda_{k}^{l}\right) /\left(\lambda_{k}^{j} \lambda_{i}^{l}\right) \mathbf{B}_{i k}^{j l}
\end{aligned}
$$

From Eq. (7) and above property of the relative homography, we can see that the matrix $\tilde{\mathbf{B}}_{i k}^{j l}$ has an eigen value $\rho_{i k}^{j l}=\left(\lambda_{i}^{j} \lambda_{k}^{l}\right) /\left(\lambda_{k}^{j} \lambda_{i}^{l}\right)$ of multiplicity two, which can be extracted directly 
from $\tilde{\mathbf{B}}_{i k}^{j l}$. Now, we can consider $\left\{\rho_{i k}^{j l}\right\}$ as alternative scale factors such that the factorization is accomplished. Assume that every canonic homographies in the measurment matrix are rescaled as $\rho_{i k}^{j l} \tilde{\mathbf{H}}_{i}^{j}=\lambda_{k}^{l} /\left(\lambda_{k}^{j} \lambda_{i}^{l}\right) \mathbf{H}_{i}^{j}$. Then, the rescaled measurement matrix can be factorized as follows:

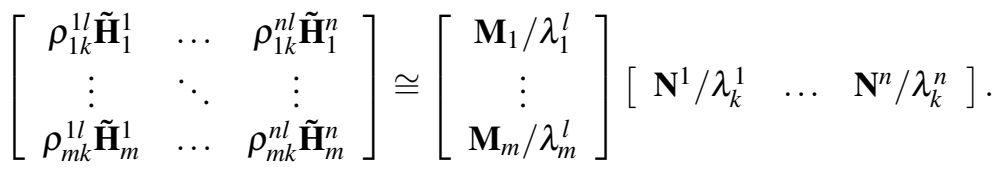

However, since the image measurements are always affected by noise, relative homography obtained above cannot has an eigen value of multiplicity two exactly. To obtain an optimal eigen value, we used the method suggested in [ $\mathbb{}[\mathbf{D}]$, in which a scalar variable $\rho$ approximating the matrix $\tilde{\mathbf{B}}_{i k}^{j l}-\rho \mathbf{I}$ to rank 1 is selected as the eigen value.

\subsection{Factorization}

From now on, it is assumed that the measurement matrix is rescaled as above. Let $\overline{\mathbf{H}}$ be the leading $3 \times 2$ submatrix of the canonic homography, which can be written as $\mathbf{\mathbf { H }}=\mathbf{K} \mathbf{R} \overline{\mathbf{S}} \mathbf{L}$. Then, the reduced measurement matrix $\overline{\mathbf{W}}$ containing all $\overline{\mathbf{H}}$ 's can be factorized as follows:

$$
\overline{\mathbf{W}} \cong\left[\begin{array}{c}
\overline{\mathbf{M}}_{1} / \lambda_{1}^{l} \\
\vdots \\
\overline{\mathbf{M}}_{m} / \lambda_{m}^{l}
\end{array}\right]\left[\begin{array}{lll}
\overline{\mathbf{N}}^{1} / \lambda_{k}^{1} & \ldots & \overline{\mathbf{N}}^{n} / \lambda_{k}^{n}
\end{array}\right]
$$

where $\overline{\mathbf{M}}_{i}=\mathbf{K}_{i} \mathbf{R}_{i}$ and $\overline{\mathbf{N}}^{j}=\overline{\mathbf{S}}^{j} \overline{\mathbf{L}}^{j}$.

As usual in the previous factorization approaches [四], [四], the SVD (Singular Value Decomposition) is used to obtain the low-rank factorization of $\overline{\mathbf{W}}$. Let the SVD of $\overline{\mathbf{W}}$ be given as:

$$
\overline{\mathbf{W}}=\mathbf{U}_{3 m \times 3 n} \mathbf{D}_{3 n \times 3 n} \mathbf{V}_{2 n \times 3 n}^{T} .
$$

Assume that the diagonal matrix $\mathbf{D}$ contains the singular values of $\mathbf{W}: \sigma_{1} \geq \sigma_{2} \geq \ldots \geq \sigma_{3 n}$. In the absence of noise, $\overline{\mathbf{W}}$ satisfying Eq. (9) has rank 3 and consequently $\sigma_{4}=\sigma_{5}=\ldots=$ $\sigma_{3 n}=0$. If noise is present, this is not the case. If we want to find the rank 3 matrix which is closest to $\overline{\mathbf{W}}$ in the Frobenius norm, such a matrix can be obtained by setting all the singular values to zero besides the three largest ones. Then, the factorization result can be given as:

$$
\begin{aligned}
\overline{\mathbf{W}}= & \overline{\mathbf{U}}_{3 m \times 3} \operatorname{diag}\left(\sqrt{\sigma_{1}}, \sqrt{\sigma_{2}}, \sqrt{\sigma_{3}}\right) . \\
& \left\{\overline{\mathbf{V}}_{2 n \times 3} \operatorname{diag}\left(\sqrt{\sigma_{1}}, \sqrt{\sigma_{2}}, \sqrt{\sigma_{3}}\right)\right\}^{T} \\
= & \hat{\mathbf{U}}_{3 m \times 3} \hat{\mathbf{V}}_{2 n \times 3}^{T} .
\end{aligned}
$$

However, the factorization result is not unique because the following is also a valid factorization:

$$
\overline{\mathbf{W}}=\left(\hat{\mathbf{U}}_{3 m \times 3} \mathbf{T}^{-1}\right)\left(\mathbf{T} \hat{\mathbf{V}}_{2 n \times 3}^{T}\right),
$$

where $\mathbf{T}$ is an arbitrary non-singular $3 \times 3$ matrix. The existence of the matrix $\mathbf{T}$ represents the non-translational part of a 3D affine ambiguity.

The results obtained up to now are equivalent to $3 \mathrm{D}$ reconstruction up to affine transformation. To resolve this affine ambiguity and upgrade the results to the metric ones, we have to impose usual self-calibration constraints and/or geometric constraints on affine reconstruction results. This issue is considered in the next section. 


\subsection{Affine and Metric Reconstruction}

From the results of section 4.2, we can say that $\overline{\mathbf{M}}_{i} \mathbf{T}=\mathbf{K}_{i} \mathbf{R}_{i} \mathbf{T}, i=1 \ldots m$ are obtained up to scale. Consider the infinite homography $\mathbf{H}_{1 i}^{\infty}$ between the first and $i$ th camera. This infinite homography can be computed from the above results as follows:

$$
\begin{aligned}
\mathbf{H}_{1 i}^{\infty} & \cong \mathbf{K}_{i} \mathbf{R}_{i} \mathbf{R}_{1}^{-1} \mathbf{K}_{1}^{-1} \\
& =\left(\overline{\mathbf{M}}_{i} \mathbf{T}\right)\left(\overline{\mathbf{M}}_{1} \mathbf{T}\right)^{-1} .
\end{aligned}
$$

The infinite homogaphis computed as in Eq. (13) for any pairs of cameras are consistent to each other because all $\overline{\mathbf{M}}_{i} \mathbf{T}$ 's are obtained simultaneously from the factorization step. Using these infinite homogaphies between the first and all the other cameras, the camera matrices of an affine reconstruction can be written as $\mathbf{P}_{1}=[\mathbf{I} \mid \mathbf{0}]$ and $\mathbf{P}_{i}=\left[\mathbf{H}_{1 i}^{\infty} \mid \mathbf{t}_{i}\right], i=2 \ldots m$. To obtain linearly the affine reconstruction of remaining $3 \mathrm{D}$ points and $\mathbf{t}_{i}$, we used the method proposed in $[\square]$ with the scene point correspondences between the views and the corresponding vertices of imaged parallelograms. In this process, since all camera positions are also obtained simultaneously, consistency of the transformations among the cameras are retained. After obtaining the affine reconstruction, we can upgrade the results to metric ones using metric constraints from scene geometry and camera intrinsic parameters [ $\square]$. For example, the orthogonality of a parallelogram's edges and zero skew of the intrinsic parameters can be used.

\section{Experimental Results}

\subsection{Simulated Experiment}

Before the experiment with real images, simulated experiments were performed in order to make careful analysis of the performance of the algorithm in various parallelogram size and singular configurations. Simulations are performed with synthetic $1024 \times 768$ images, taken by three cameras with the following intrinsic parameters: $\left(f_{u}, f_{v}, s, u_{0}, v_{0}\right)=(1200,1000,0$, $512,384)$. Two squares were placed in front of the three cameras. The cameras were placed in a row and the distance between close cameras were $1 \mathrm{~m}$. Zero-mean uniformly-distributed noise over the interval [- 0.5 pixel, 0.5 pixel] was added to the projections. The constraints used in this experiment were: orthogonality of the edge of the squares and zero skew of the cameras.

First, we test the performance while varying the edge size of the squares from $0.5 \mathrm{~m}$ to $1.5 \mathrm{~m}$. In this experiments, the angle between the planes of the two squares were set to $120^{\circ}$. Fig. 3(a) shows the results. We can see that the algorithm can acquire reasonable results for the edge size over $0.8 \mathrm{~m}$.

Second, we test the performance while varying the angle between the planes of the two squares from $50^{\circ}$ to $170^{\circ}$. In this experiments, the edge size of the squares were set to $1 \mathrm{~m}$. Fig. 3(b) shows the results. We can see that the singular configurations occur when the angles are near to $50^{\circ}$ and $170^{\circ}$. In case of $50^{\circ}$, one of the squares are too tilted to the viewing direction of one of the cameras. In case of $170^{\circ}$, two squares are on an identical plane. 


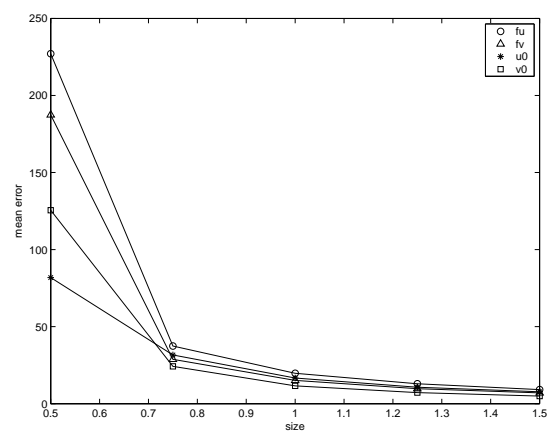

(a)

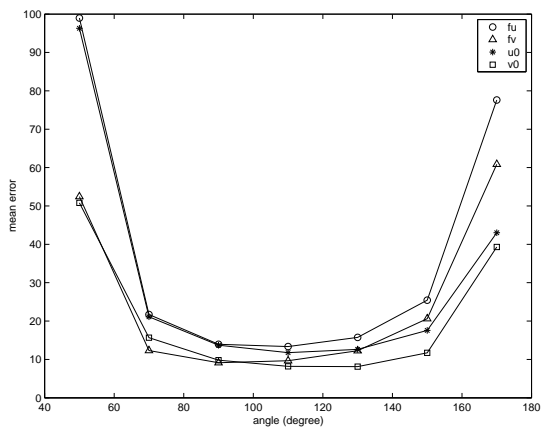

(b)

Figure 3: The results from the simulated experiments for analyzing the relation between the performance and (a) Size of parallelograms. (b) Angle between the planes.

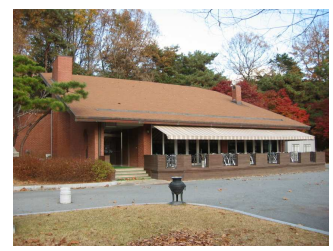

(a)

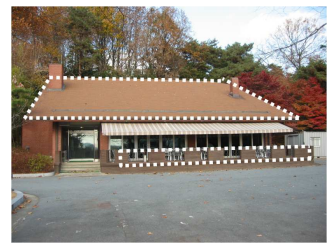

(b)

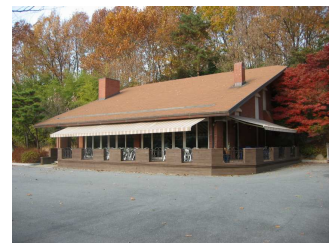

(c)

Figure 4: Three captured images for House Scene experiment. Image (a) 1, (b) 2, (c) 3

\subsection{Real Image Experiment - House Scene}

The resolution of the images was $853 \times 640$ and the cameras were not static. Three captured images are shown in Fig. 4. In this example, since there are no parallelepiped of which the six vertices can be seen across the images, the previous method [ख] cannot be used. The two parallelograms denoted in Fig. 4(b) with the white dotted lines were used as the input for the proposed algorithm. The lines corresponding to the dotted lines were extracted and the vertices of parallelograms were obtained from the intersections of the lines. Metric constraints used in this experiment were: orthogonality of the edge of the building, unit aspect ratio and zero skew of cameras. Fig. 5 shows the reconstructed model and the camera pose in new view positions.

\subsection{Real Image Experiment - Pyramid Scene}

The resolution of the images was $1024 \times 768$ and the camera parameters were static while the images were captured. Three captured images are shown in Fig. 6. The two parallelograms denoted in Fig. 6(a) with the white dotted lines were used as the input for the algorithms. The vertices of parallelograms were obtained using the same method in Section 5.2. Metric constraints used in this experiment were: static intrinsic parameters, zero skew of cameras. Fig. 7 shows the reconstructed model and the camera pose in new view positions. 


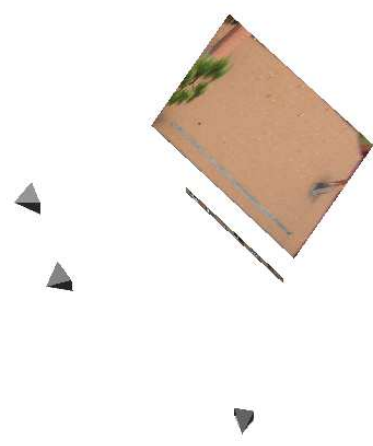

(a)

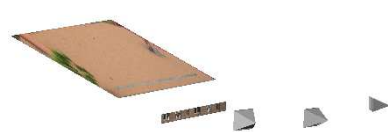

(b)

Figure 5: Reconstructed model and camera pose for House Scene experiment.

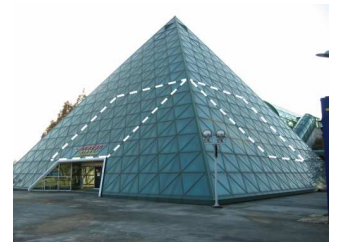

(a)

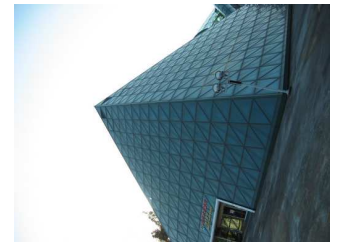

(b)

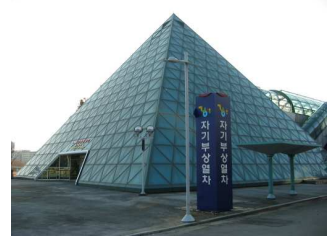

(c)

Figure 6: Three captured images for Pyramid Scene experiment. Image (a) 1, (b) 2, (c) 3

\section{Conclusion and Future works}

In this paper, a novel framework was proposed for camera calibration and scene modeling when there are parallelograms in a scene. The proposed method was based on a factorizationbased approach for obtaining the consistency of rigid transformations among cameras. It was shown that it is possible to factorize the measurement matrix composed of canonic planar homographies into the camera and plane parameters. It was also shown from the real image experiments that using geometric constraints embedded in the parallelograms enables more stable calibration results to be obtained and allows the tasks to be performed with fewer images. However, there is future research topic which remain unresolved here. In the formulation of this paper, it is assumed that all parallelograms are viewed in all images. One of the usual problems for the factorization-based approach is to handle missing data. If some parallelograms are occluded in some images, some entries of measurement matrix cannot be obtained. We did not handle this problem in this paper but a great deal of effort in different type of factorization-based approaches can be referred [], [四].

Acknowledgment This work was supported by the strategic technology development program of MCST/MKE/KEIT. [KI001798, Development of Full 3D Reconstruction Technology for Broadcasting Communication Fusion] 


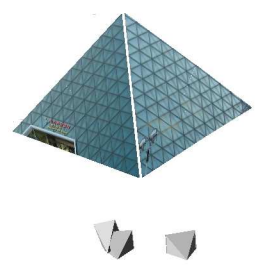

(a)

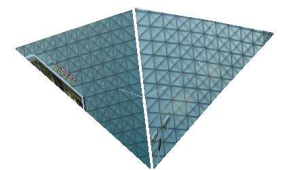

(b)

Figure 7: Reconstructed model and camera pose for Pyramid Scene experiment.

\section{References}

[1] Lourdes Agapito, E. Hayman, and I. Reid. Self-calibration of rotating and zooming cameras. International Journal of Computer Vision, 45(2):107-127, November 2001.

[2] R. Cipolla, T. Drummond, and D. P. Robertson. Camera calibration from vanishing points in images of architectural scenes. In Proc. British Machine Vision Conferece, pages 382-391, Nottingham, England, September 1999.

[3] Pär Hammarstedt, Fredrik Kahl, and Anderson Heyden. Affine reconstruction from translational motion under various autocalibration constraints. Journal of Mathematical Imaging and Vision, 24(2):245-257, March 2006.

[4] Richard Hartley and Andrew Zisserman. Multiple View Geometry in Computer Vision, Second Edition. Cambridge University Press, Cambridge, 2003.

[5] Davis Jacobs. Linear fitting with missing data: Applications to structure from motion and to characterizing intensity images. In Proc. IEEE International Conference on Computer Vision and Pattern Recognition, pages 206-212, San Juan, Puerto Rico, June 1997.

[6] David Jelinek and Camillo J. Taylor. Reconstruction of linearly parameterized models from single images with a camera of unknown focal length. IEEE Trans. Pattern Anal. Machine Intell., 23(7):767-773, July 2001.

[7] Jae-Hean Kim. Linear stratified approach for 3D modelling and calibration using full geometric constraints. In Proc. IEEE International Conference on Computer Vision and Pattern Recognition, pages 2144 - 2151, Miami, FL, USA, June 2009.

[8] Jae-Hean Kim and Byung Tae Choi. Estimation of camera parameters from arbitrary parallelograms. In Proc. IEEE/RSJ International Conference on Intelligent Robots and Systems, pages 3597-3603, Nice, France, September 2008.

[9] JaeChul Kim, Kyoung Mu Lee, and Sang Uk Lee. Camera calibration using a single arbitrary parallelogram. In Proc. Asian Conference on Computer Vision, Jeju Island, Korea, January 2004. 
[10] David Liebowitz and Andrew Zisserman. Combining scene and auto-calibration constraints. In Proc. IEEE International Conference on Computer Vision, pages 293-300, Kerkyra, Greece, September 1999.

[11] Ezio Malis and Roberto Cipolla. Camera self-calibration from unknown planar structures enforcing the multiview constraints between collineations. IEEE Trans. Pattern Anal. Machine Intell., 24(9):1268-1272, September 2002.

[12] Daniel Martinec and Tomáš Pajdla. Structure from many perspective images with occlusions. In Proc. European Conference on Computer Vision, pages 355-369, Copenhagen, Denmark, May 2002.

[13] T. Moons, L. Van Gool, M. Proesmans, and E. Pauwels. Affine reconstruction from perspective image pairs with a relative object-camera translation in between. IEEE Trans. Pattern Anal. Machine Intell., 18(1):77-83, January 1996.

[14] Marc Pollefeys and Luc Van Gool. Stratified self-calibration with the modulus constraint. IEEE Trans. Pattern Anal. Machine Intell., 21(8):707-724, August 1999.

[15] Marc Pollefeys, Luc Vav Gool, Maarten Vergauwen, Frank Verbiest, Kurt Cornelis, Jan Tops, and Reinhard Koch. Visual modeling with a hand-held camera. International Journal of Computer Vision, 59(3):207-232, October 2004.

[16] Carsten Rother and Stefan Carlsson. Linear multi view reconstruction and camera recovery using a reference plane. International Journal of Computer Vision, 49(2-3): 117-141, September 2002.

[17] Carsten Rother, Stefan Carlsson, and Dennis Tell. Projective factorization of planes and cameras in multiple views. In Proc. International Conference on Pattern Recognition, pages 737-740, Quebec, Canada, August 2002.

[18] Peter Sturm. Algorithms for plane-based pose estimation. In Proc. IEEE International Conference on Computer Vision and Pattern Recognition, pages 706-711, Hilton Head Island, SC, USA, June 2000.

[19] C. Tomasi and T. Kanade. Shape and motion from image streams under orthogrphy: a factorization method. International Journal of Computer Vision, 9(2):137-154, November 1992.

[20] Toshio Ueshiba and Fumiaki Tomita. Plane-based calibration algorithm for multicamera systems via factorization of homography matrices. In Proc. IEEE International Conference on Computer Vision, pages 966-973, Nice, France, October 2003.

[21] Marta Wilczkowiak, Peter Sturm, and Edmond Boyer. Using geometric constraints through parallelepipeds for calibration and 3D modelling. IEEE Trans. Pattern Anal. Machine Intell., 27(2):194-207, February 2005.

[22] F. C. Wu, F. Q. Duan, and Z. Y. Hu. An affine invariant of parallelograms and its application to camera calibration and 3D reconstruction. In Proc. European Conference on Computer Vision, pages 191-204, May 2006.

[23] Lihi Zelnik-Manor and Michal Irani. Multiview constraints on homographies. IEEE Trans. Pattern Anal. Machine Intell., 24(2):214-223, February 2002. 